\title{
Distinct roles of integrins $\alpha 6$ and $\alpha 4$ in fetal liver hematopoietic stem and progenitor cell homing
}

Hong Qian ${ }^{1}$, Elisabeth Georges-Labouesse ${ }^{2}$, Alexander Nyström ${ }^{3}$, Anna Domogatskaya ${ }^{4}$, Karl Tryggvason ${ }^{4}$, Sten Eirik W Jacobsen ${ }^{1}$, Marja Ekblom ${ }^{1}$

${ }^{1}$ Hematopoietic Stem Cell Laboratory, Lund Strategic Research Center for Stem Cell Biology and Cell Therapy, Lund University, 22184 Lund, Sweden; ${ }^{2}$ The Institute of Genetics and Molecular and Cellular Biology, BP 10142, 67400, Illkirch, France. ${ }^{3}$ Department of Cell and Matrix Biology, Lund University, Lund, Sweden; ${ }^{4}$ Department of Medical Biochemistry and Biophysics, Karolinska Institute, Stockholm, Sweden

Efficient homing of hematopoietic stem cells (HSC) in bone marrow (BM) is a prerequisite for establishment of hematopoiesis during development and following transplantation. However, the cellular and molecular pathways that control the homing of HSCs, in particular, of fetal HSCs are still not well understood. Of integrin receptors, the role of integrin $\alpha 4$ receptor during embryonic hematopoiesis has been established by using gene ablated chimeric mice and function blocking antibodies. However, the specific function of integrin $\alpha 4$ in homing of fetal liver HSPC remains less well defined. We have previously found that integrin $\alpha 6$ mediates human hematopoietic stem and progenitor cell (HSPC) adhesion to and migration on its specific ligands, laminin-8 and laminin-10/11 in vitro (Gu et al, Blood, 2003; 101:877). Furthermore, integrin $\alpha 6$ is required for adult mouse HSC homing to BM in vivo (Qian et al., Blood, 2006; 107:9). We have found that the integrin $\alpha 6$ and $\alpha 4$, like in adult HSCs, is ubiquitously ( $>99 \%$ ) expressed also in fetal liver HSPCs (lin-Sca-1+c-Kit+, LSK ). Here we studied the contribution of $\alpha 6$ and $\alpha 4$ integrins in homing of fetal liver hematopoietic cells into adult BM by using function-blocking antibodies and an integrin $\alpha 6$ knockout mouse model. We found an ubiquitous expression of integrin $\alpha 6$ on fetal liver LSK HSPCs. Genetic ablation of integrin $\alpha 6$ resulted in reduced homing of fetal liver progenitors to BM of lethally irradiated adult recipients. In agreement with this, the integrin $\alpha 6$ antibody inhibited homing of fetal liver progenitors into BM and spleen. The role of integrin $\alpha 6$ in homing and engraftment of fetal liver HSCs was studied by a competitive repopulation assay by using integrin $\alpha 6-/-$ or $\alpha 6+/+$ fetal liver cells. Absence of $\alpha 6$ integrin in fetal liver cells did not cause any engraftment defect as compared to wildtype cells. Consistent with this, anti-integrin $\alpha 6$ antibody did not either reduce BM homing of shortterm or long-term HSCs. In contrast, homing of fetal liver HSCs and HPCs to BM was virtually abrogated after treatment with integrin $\alpha 4$ antibody. These results show that $\alpha 6$ integrin acts as a homing receptor during fetal liver progenitor cell homing to $\mathrm{BM}$, whereas homing and engraftment of multilineage repopulating HSCs is independent of $\alpha 6$ integrin function. In contrast, integrin $\alpha 4$ receptor is crucial for homing of all HSPCs, indicating distinct roles for each integrin during fetal hematopoieisis. (Qian, et al., 2006, ASH abstract, Qian et al., Blood, 2007 in press)

Keywords: integrin, homing, bone marrow, hematopoietic stem cell

Cell Research (2008) 18:s101. doi: 10.1038/cr.2008.191; published online 4 August 2008

Correspondence: Marja Ekblom

E-mail: Marja.Ekblom@med.lu.se

Short description of Hong Qian, PhD is currently working as a postdoctoral researcher in Hematopoietic Stem Cell Laboratory, Lund Stem Cell Center, Lund University, in Sweden. I was since 2003 studying as a Ph D student in Lund Stem Cell Center, Lund University under supervision of Dr Marja
Ekblom and Sten Eirik Jacobsen.I obtained my doctoral degree in Lund University in June 2007. My research projects have been focusing on 'Bone marrow niche components on regulation of hematopoietic stem and progenitor cells. In particular, the role of extracellular matrix protein lamimins and growth factor thrombopoietin in migration and development of hematopoietic stem and progenitor cells'. 